\title{
Es gibt mehr Experten als Patienten
}

Es gibt Handlungsempfehlungen verschiedener Fachgesellschaften für die Betreuung von Rheuma-Patienten in Verbindung mit Covid-19, evidenzbasierte Daten gibt es bislang noch nicht und kann es eigentlich auch nicht geben. Prim. Doz. Dr. Burkhard Leeb, Leiter der 2. medizinischen Abteilung des Landesklinikums Weinviertel Stockerau und des Institutes für Klinische Rheumatologie der Karl Landsteiner Gesellschaft, Hollabrunn, im Gespräch über Auswirkungen von COVID-19 speziell auf Rheumatiker und den Einfluss von Antirheumatika auf die Virus-Infektion.

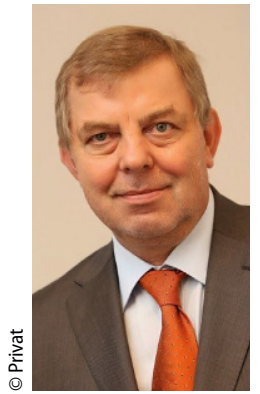

Prim. Doz. Dr. Burkhard F. Leeb
Sind Rheuma-Patienten besonders anfällig an COVID-19 zu erkranken? Leeb: Grundsätzlich, das ist unbestritten, sind Patienten mit Autoimmunerkrankungen natürlich infektanfälliger. Was COVID-19 betrifft ist es schwierig klare Aussagen zu treffen, da die Erkrankung in Österreich eine Prävalenz von etwa 0,18 hat - das ist an der Grenze zu einer Orphan disease. Und auch entzündlich-rheumatische Erkrankungen sind selten, großzügig gerechnet haben sie eine Prävalenz von zwei Prozent. Daher ist ein Zusammentreffen beider Erkrankungen bei diesen Prävalenzen relativ unwahrscheinlich.

In Studien kommen muskuloskelettale Erkrankungen als Begleiterkrankungen praktisch nicht vor. Was die internationalen RheumaRegister allerdings zeigen ist, dass mehr Frauen als Männer an COVID-19 erkranken. Das steht im Gegensatz zur übrigen Bevölkerung, wo eher Männer als Frauen an COVID-19 erkranken. Davon abgesehen zeigen die Daten der Register keine Abweichungen zur Normalpopulation.

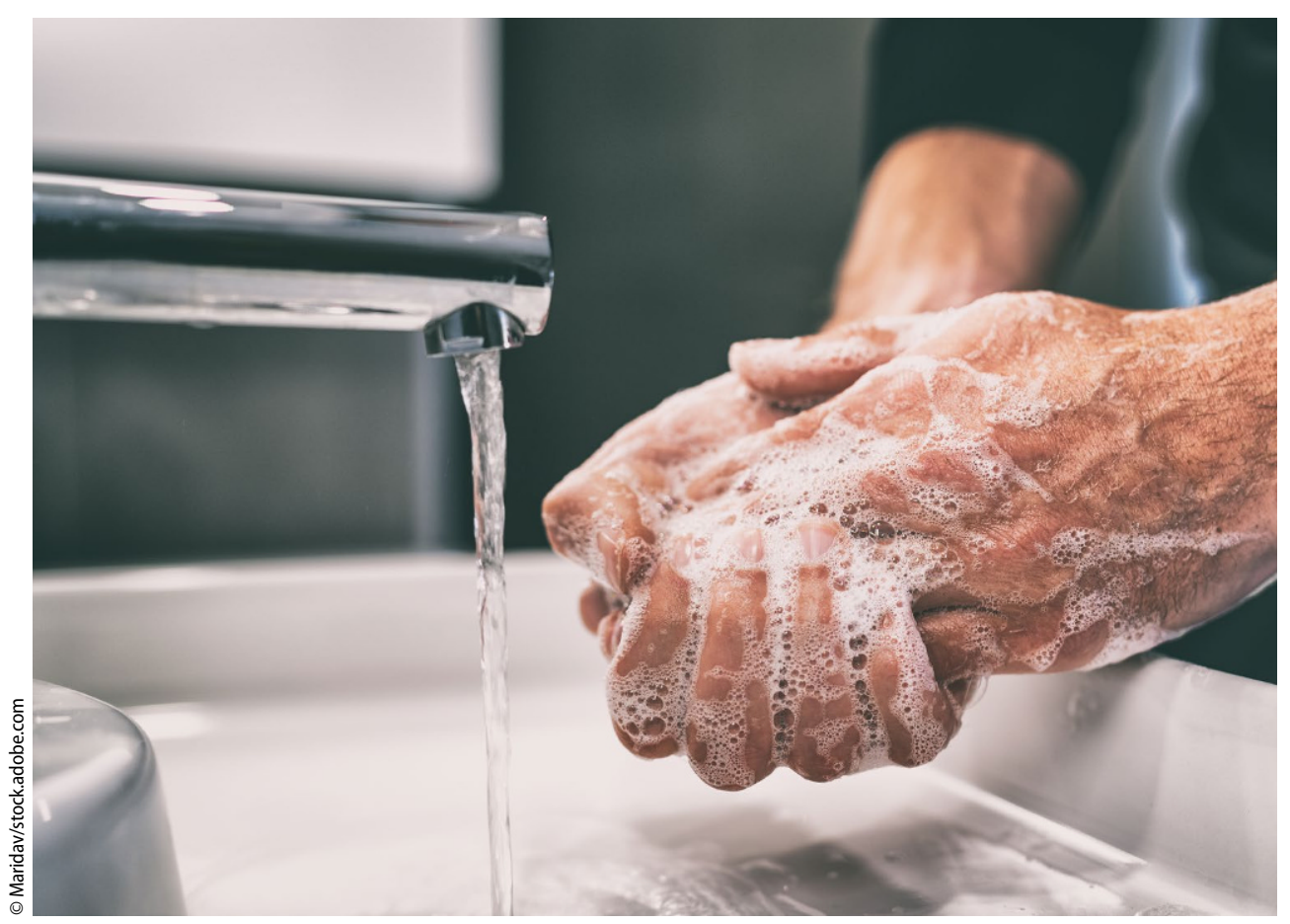

\ Distanz halten und Hände waschen sind in jedem Fall gesundheitsförderliche Maßnahmen
Wie viele an COVID-19 erkrankte Patienten wurden bisher im österreichischen Biologika-Register erfasst?

Leeb: Im österreichischen Biologika-Register wurden bisher zwei COVID-19-Patienten erfasst, beide sind Spondyloarthritis-Patienten unter Therapie mit TNF-Blockern. Mit der COVID-19-Infektion wurden die TNF-Blocker pausiert. Soweit ich weiß, haben sich beide Patienten bereits erholt und erhalten inzwischen wieder ihre übliche RheumaTherapie.

Gibt es Daten zu Aufenthalten auf Intensivstationen von Rheuma-Patienten, die an COVID-19 erkranken?

Leeb: Daten zu Aufenthalten auf Intensivstationen und zum Outcome von Rheuma-Patienten mit COVID-19 gibt es kaum.

Zahlen der COVID-19 Global Rheumatology Alliance zeigen, dass von 872 Patienten 79 - also $9 \%$ - verstorben sind. Das würde einer höheren Todesrate als in der Normalbevölkerung entsprechen, aber solche Vergleiche sind derzeit kaum möglich und eigentlich auch nicht zulässig. $45 \%$ der in dieses internationale Register aufgenommenen Rheuma-Patienten wurden hospitalisiert. $67,78 \%$ wurden wieder gesund, 21,33\% sind nicht gesundet und bei 95 Patienten ist der Outcome unbekannt.

Gefühlt könnte eine COVID19-Infektion also schwerer verlaufen als in der Normalbevölkerung, aber eine zuverlässige 
Aussage ist aufgrund dieser Daten noch nicht möglich.

Was empfehlen Sie Ihren Patienten? Leeb: In jedem Fall ist die gute Behandlung der Autoimmunerkrankung ein Schutz vor Infektanfälligkeit: Je besser behandelt, umso geringer die Infektanfälligkeit. Das gilt übrigens auch für Hypertonie, Diabetes etc.

》) Rauchen scheint kein Risikofaktor für einen schweren Verlauf von COVID-19 zu sein

Tritt eine Infektion mit SARS-CoV-2 auf, empfiehlt sich dasselbe Vorgehen wie bei jeder anderen Infektionskrankheit: ein Unterbrechen der Therapie - von Medikament zu Medikament ist das sehr unterschiedlich zu handhaben - bei Auftreten von Infektsymptomen.

Ich würde Malariamedikamente nicht unterbrechen, ich würde auch niedrig dosiertes Kortison bis etwa $10 \mathrm{mg}$ nicht unterbrechen. Aber MTX, auch Leflunomid, Biologika- und JAK-Kinase-Therapien würde ich im Fall eines Infektes pausieren. Ist der Infekt abgeklungen, soll die Behandlung in der vorher durchgeführten Form fortgesetzt werden.

Das wird übrigens auch bei z.B. Herpes zoster und Influenza so gehandhabt. wie auch bei bakteriellen Infekten. Bei Harnwegsinfekten mit Fieber, bei Bronchitiden etc. wird ebenso vorgegangen.

Welche Bedeutung spielen Komorbiditäten wie Hypertonie, COPD, Diabetes und Adipositas bei RheumaPatienten?

Leeb: Die Komorbiditäten bei rheumatologischen Patienten sind, wenn man die Daten der COVID-19 Global Rheumatology Alliance betrachtet, in etwa so verteilt wie in der Normalbevölkerung. 628 der im Register erfassten Patienten sind Europäer, die restlichen etwa 250 Patienten stammen aus anderen Erdteilen. Etwas mehr als ein Viertel der erfassten RheumaPatienten leidet außerdem an Hypertonie, etwas weniger als ein Viertel an COPD, etwa $16 \%$ an KHK, $15 \%$ an Diabetes. Acht Prozent sind sehr dick, mit einem BMI über 40. Sieben Prozent der Patienten haben eine renale Insuffizienz und sechs Prozent ein Karzinom. Das entspricht in etwa auch den Daten der nationalen Register zu den Begleiterkrankungen.

Auch die Altersverteilung ist die übliche: es sind relativ wenig junge Rheumatiker erfasst, was auch dem Krankheitsauftreten entspricht. Vor allem Patienten zwischen 30 und 65 Jahren scheinen in den Daten der COVID-19 Global Rheumatology Alliance auf, etwas mehr als ein Viertel der Patienten ist mehr als 65 Jahre alt.

Mit der einen Ausnahme also, dass im Gegensatz zur übrigen Bevölkerung mehr Frauen mit Rheuma von COVID-19 betroffen sind - was dem Verteilungsmuster der rheumatischen Erkrankungen entspricht - zeigen die Daten keine besonderen Auffälligkeiten.

Welche Rolle spielen Nikotinabusus, Steroideinnahme und Alter für die Infektanfälligkeit?

Leeb: Das Sterberisiko steigt ab der Geburt - je älter jemand wird, umso näher ist er dem Tod. Wenn der Tod per se und nicht die verkürzte Lebenserwartung als Outcome gewertet wird, ist das Alter ein selbsterklärender Risikofaktor. Spannend ist, dass die Todesraten von COVID-19-Patienten international rascher absinken als die Fallzahlen. Das könnte bedeuten, dass die COVID-Infektion manchmal das Tüpfelchen auf dem „i“ darstellt und das Ableben herbeiführt - wie das auch bei der Grippe und anderen Infekten der Fall ist.
Zu Steroiden ist zu sagen, dass höhere Dosierungen die Infektanfälligkeit nachgewiesenermaßen erhöhen. Gleichzeitig sagen manche Empfehlungen, dass niedrig dosiertes Kortison für kurze Zeit zur Behandlung von COVID-19 eingesetzt werden kann. Daraus leitet sich für mich auch ab, niedrig dosiertes Kortison in der RheumaTherapie nicht zu unterbrechen.

》) Wer zu einer bestimmten Zeit nicht an COVID-19 erkrankt war, war gar nicht krank

Rauchen wird kontroversiell diskutiert. Bei den hospitalisierten Patienten in China, wo sehr viele Menschen rauchen, ist der Anteil der Raucher geringer als erwartet. Weniger Raucher wurden schwer krank als Nichtraucher. Es gibt eine Hypothese, die besagt, dass Nikotin über das cholinerge antiinflammatorische System entzündungshemmend wirken könnte. Rauchen scheint also kein Risikofaktor für einen schweren Verlauf von COVID-19 zu sein. Aufgrund der anderen, mit dem Rauchen assoziierten Komorbiditäten, ist Rauchen natürlich trotzdem nicht zu empfehlen.

Einige in der Rheumatologie eingesetztePräparate werden zur Therapie von COVID-19 getestet. Könnten sie Rheumatiker vor COVID-19 "schützen"?

Leeb: Ich glaube nicht, dass Medikamente Rheumapatienten vor einer Infektion mit SARS-CoV-2 schützen. Hier erinnert die Debatte an die Virushypothese bei rheumatischen Erkrankungen. Die Virushypothese geistert seit langem in der Rheumatologie herum: Alle paar Jahre wurde ein Virus verdächtigt chronisch-entzündliche Erkrankungen auszulösen.

Tatsache ist, dass es offensichtlich bei schweren Verläufen von 


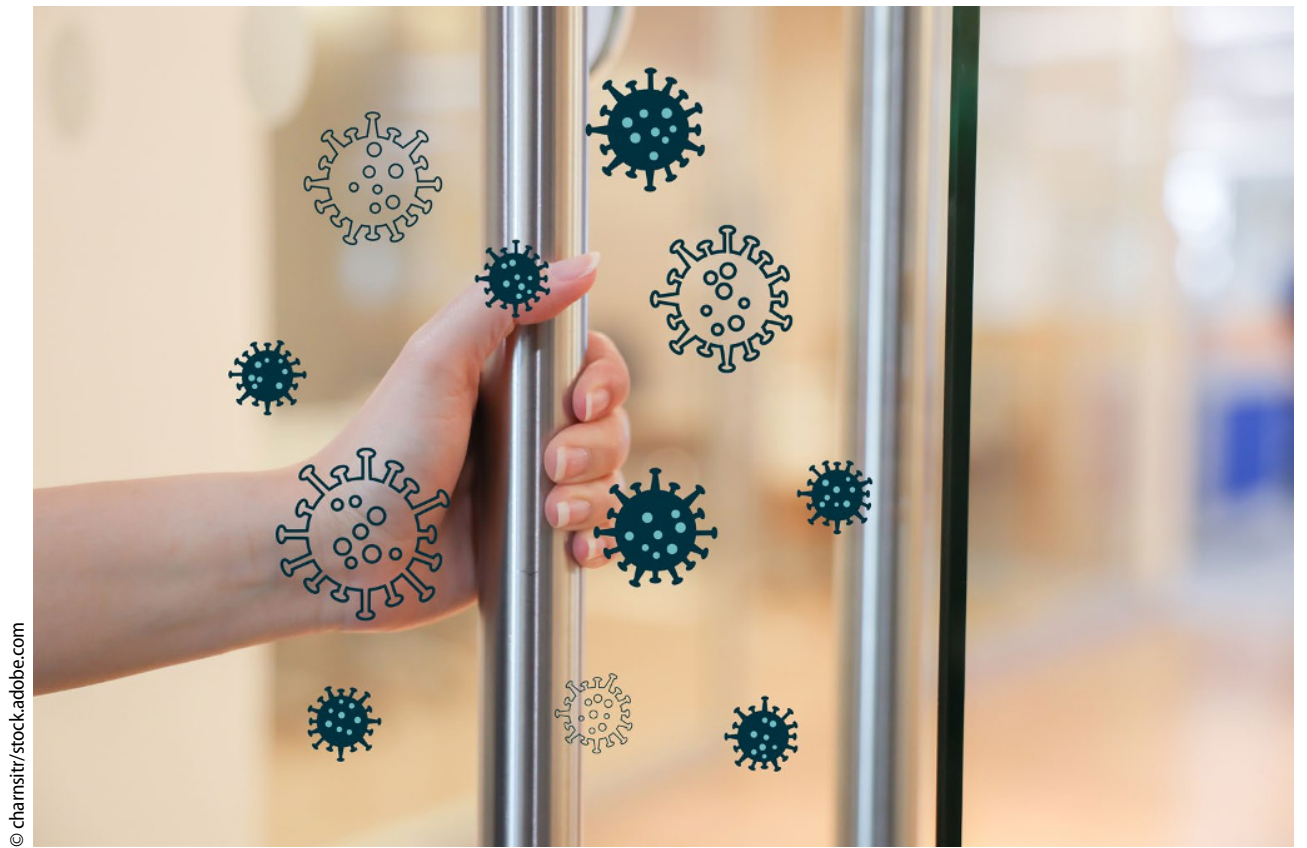

\ Sich nicht bei jeder Gelegenheit um den Hals zu fallen, ist zu Infektionszeiten auch vernünftig

COVID-19 zu einer Aktivierung der inflammatorischen Kaskade und dem so genannten Zytokinsturm kommt. Diesen Zytokonsturm versucht man jetzt zu blockieren, indem pro-inflammatorische Zytokine durch die IL6-Rezeptor-Antagonisten Tocilizumab oder Sarilumab und den JAK-Inhibitor Baricitinib gehemmt werden. Tocilizumab ist meines Wissens in China mittlerweile zur Therapie der schweren COVID-Infektion zugelassen.

Hydroxychloroquin hat sich nicht wirklich bewährt. In einer Studie mit $600 \mathrm{mg} / \mathrm{Tag}$ - das ist ein Drittel mehr als die in der Rheumatologie eingesetzte Normaldosierung - war Hydroxychloroquin in Kombination mit Azithromycin für das Herz nicht unbedingt gesund - im Sinne gehäufter Rhythmusstörungen. Die Studie musste abgebrochen werden.

Auch andere Medikamente aus der rheumatologischen Basistherapie wurden in der Behandlung von COVID-19 getestet: Methotrexat und sogar Auranofin und auch Anakinra, denn auch IL1 ist ein Schlüsselzytokin in der proinflammatorischen Kaskade.

Das bedeutet insgesamt nicht, dass Rheuma-Patienten durch die Therapie geschützt sind, aber es ist ein zusätzliches Argument ihre Therapie nicht zu unterbrechen.

Wie sieht es mit der Verfügbarkeit von Rheuma-Medikamenten in Österreich aus?

Leeb: Orales Methotrexat ist schlecht verfügbar, das war aber schon vor COVID-19 so. Hydroxychloroquin wird jetzt relativ einfach verfügbar sein, weil es zum Einsatz gegen COVID-19 nicht besonders geeignet erscheint. Die Österreichische Gesellschaft für Rheumatologie hat außerdem mit der Apothekerkammer eine Übereinkunft getroffen, damit Patienten, die Hydroxychloroquin aufgrund eines bestehenden Lupus erythematodes erhalten, damit versorgt werden.

Wie beurteilen Sie persönlich die aktuelle Entwicklung rund um COVID-19?
Leeb: Interessant ist, dass die Evidenz derzeit eine untergeordnete bis gar keine Rolle mehr spielt. Einerseits gibt es wenig Evidenz, andererseits wird auch nicht mehr darauf geachtet. Dass Distanz halten und Hände waschen gesundheitsförderlich sind, ist ein Allgemeinplatz. Ich frage mich, wie oft sich die Menschen sonst ihre Hände gewaschen haben? Sich nicht bei jeder Gelegenheit um den Hals zu fallen und Menschenansammlungen zu meiden, ist zu Infektionszeiten auch vernünftig.

Alle anderen Maßnahmen sind in Österreich etwas geheimnisumwittert und muten fast okkultistisch an, weil die Entscheidungsgrundlagen im Dunklen bleiben. Ich fände es vernünftig, die Entscheidungsgrundlagen, welche auch immer das sind, offen zu legen. Ich persönlich wundere mich, wie viele Experten es gibt - man muss hier den Kabarettisten Lukas Resetarits zitieren: Es gibt mehr Experten als Patienten. Denn mittlerweile gibt es Gott sei Dank fast nur mehr 1000 Kranke und nahezu so viele Experten.

\) Vieles wurde im Zuge von COVID-19 emotionalisiert. Man sieht, was aus der Angst alles entstehen kann

Ich denke, die Beurteilung der Maßnahmen wird erst zu einem späteren Zeitpunkt möglich sein. Anzunehmen ist auch, dass zu keinem Zeitpunkt mehr als 0,1\% der österreichischen Bevölkerung, das wären etwa 9000 Menschen, gleichzeitig erkrankt waren. Man könnte, rein nach Zahlen, auch formulieren, dass COVID-19 einen gesundheitsförderlichen Aspekt besitzt: In Italien gab es nur halb so viele Herzstillstände in den Spitälern wie sonst, in den Niederlanden kam es zu einer deutlichen Reduktion der onkologischen Diagnosen, 
in Österreich gab es $30 \%$ weniger Herzinfarkte und auch weniger gastrointestinale Blutungen. Man könnte also glauben, wer zu einer bestimmten Zeit nicht an COVID-19 erkrankt war, war gar nicht krank. Ich denke aber, dass diese Reduktionen darauf zurückzuführen sind, dass viele Menschen die Spitäler oder auch niedergelassenen Ärzte nicht aufgesucht bzw. nicht erreicht haben, denn Tatsache ist auch, dass einige Krankenhäuser zu den Hochzeiten von COVID-19 geringer belegt waren, als sonst zu Weihnachten.

Auch die Verwendung von Kapazitäten im Gesundheitsbereich wird derzeit deutlich. Wer einige GrippeSaisonen als Spitalsarzt miterlebt hat, fragt sich schon, wie bei 3500 vorhandenen Intensivbetten 300 intensivpflichtige Patienten ein Problem darstellen können? Eng- pässe gab es auch früher schon man erinnere sich an die immer wiederkehrende GangbettenDiskussion - und auch Entscheidungen, wie ein Patient weiter behandelt wird, waren immer zu treffen.

Ich bin absolut dafür, vorsichtig zu sein, aber Vieles wurde im Zuge von COVID-19 emotionalisiert, und man sieht, was aus der Angst alles entstehen kann. Und, ich vermisse in den Krisenstäben neben Mathematikern und Systemanalytikern, vielleicht zu Unrecht, eine größere Anzahl von Menschen, die in den letzten Jahren mit direkten medizinischen Problemen tatsächlich zu tun hatten, daher würde ich mir eine Bekanntgabe der Beraterstäbe wünschen.

Das Gespräch führte Mag. Tanja Fabsits am 14. Mai 2020

\section{Weitere Informationen:}

COVID-19 Global Rheumatology Alliance: https://rheum-covid.org

EULAR COVID-19-Datenbasis: https://bit.ly/3bOfW19

Österreichisches Biologika-Register: https://www.bioreg.at/covid-19/

Österreichische Gesellschaft für Rheumatologie und Rehabilitation: https://rheumatologie.at/ gesellschaft/covid-19/

Hinweis des Verlags. Der Verlag bleibt in Hinblick auf geografische Zuordnungen und Gebietsbezeichnungen in veröf-

fentlichten Karten und Institutsadressen neutral.

rheuma plus $2020 \cdot 19: 84-87$

https://doi.org/10.1007/s12688-02000348-0

(c) Springer-Verlag GmbH Austria, ein Teil von Springer Nature 2020

Hier steht eine Anzeige. 